\title{
Fosfitos para o controle de podridões pós-colheita em maçãs 'Fuji' durante o armazenamento refrigerado
}

\author{
Use of phosphites for rot control during cold storage of 'Fuji' apples
}

\author{
Auri Brackmann ${ }^{1}$ Ricardo Fabiano Hettwer Giehl ${ }^{2}$ \\ Ivan Sestari $^{3}$ Cristiano André Steffens ${ }^{4}$
}

\section{RESUMO}

Foi conduzido um experimento com o objetivo de avaliar o efeito da aplicação de adubos foliares contendo fosfitos de potássio e fosfitos de cálcio mais boro sobre o controle de podridões em maçãs 'Fuji' durante a frigoconservação. $O$ delineamento experimental utilizado foi o inteiramente casualizado com quatro repetições de 25 frutos. Foram realizadas quatro lesões distribuídas de forma eqüidistante na região equatorial dos frutos, com $3 \mathrm{~mm}$ de diâmetro e $5 \mathrm{~mm}$ de profundidade. Os frutos foram submersos em uma solução com suspensão de esporos, onde permaneceram por 20 segundos. Após, foram aplicados os trata-

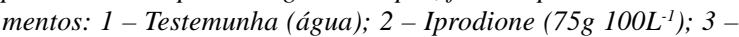
cloreto de cálcio $\left(\mathrm{CaCl}_{2}\right)$ a 2\%; 4 - fosfito de potássio $(250 \mathrm{~mL}$ $\left.100 L^{-1}\right)+\mathrm{CaCl}_{2}(2 \%) ; 5$-fosfito de potássio $\left(250 \mathrm{~mL} 100 \mathrm{~L}^{-1}\right) ; 6-$ fosfito de cálcio mais boro $\left(300 \mathrm{~mL} 100 \mathrm{~L}^{-1}\right)$; e 7 -fosfito de potássio (250mL 100L $\left.\mathrm{L}^{-1}\right)$ na suspensão de esporos por uma hora. Os frutos foram armazenados em ambiente refrigerado à temperatura de $0^{\circ} \mathrm{C}$ durante 14 dias. As avaliações foram realizadas na saída da câmara e aos 2, 4, 6 e 8 dias a $20^{\circ} \mathrm{C}$. Verificou-se que os frutos tratados com fosfito de potássio $\left(250 \mathrm{~mL} 100 \mathrm{~L}^{-1}\right)+\mathrm{CaCl}_{2}$ (2\%) apresentaram a menor incidência de podridões e o menor diâmetro de lesão. Esses resultados foram semelhantes aos obtidos com a aplicação do fungicida padrão Iprodione e superiores à aplicação de fosfito de potássio isoladamente. $O$ uso de fosfito de cálcio mais boro não foi eficiente.

Palavras-chave: Malus domestica Borkh., adubos foliares, Penicillium spp.

\section{ABSTRACT}

This experiment aimed to evaluate the effectiveness of potassium $(\mathrm{K})$ phosphite and calcium $(\mathrm{Ca})+$ borum $(\mathrm{B})$ phosphite to control rot during cold storage of 'Fuji' apples. Four equidistant lesions were made in the central region of the fruits, measuring $5 \mathrm{~mm}$ wide and $3 \mathrm{~mm}$ deep. After the treatments, fruits were submerged for 20 seconds in a spore suspension, except for the last treatment, in wich the immersion lasted one hour. The treatments were as follow: 1. control (water); 2. fungicide Iprodione $\left(75 \mathrm{~g}^{100 \mathrm{~L}^{-1}}\right)$; 3. $\mathrm{CaCl}_{2}(2 \%) ; 4$. potassium phosphite $(250 \mathrm{~mL} 100 \mathrm{~L}$ $\left.{ }^{1}\right)$ plus $\mathrm{CaCl}_{2}(2 \%)$; 5. potassium phosphite $\left(250 \mathrm{~mL} 100 \mathrm{~L}^{-1}\right) ; 6 . \mathrm{Ca}$ + B phosphite $\left(300 \mathrm{~mL} 100 \mathrm{~L}^{-1}\right)$; and 7. potassium phosphite $(250 \mathrm{~mL}$ $\left.100 L^{-1}\right)$ in spore suspension for one hour. Fruits were refrigerated at $0^{\circ} \mathrm{C}$ during 14 days and evaluated at removal from cold room and after 2, 4, 6 and 8 days at $20^{\circ} \mathrm{C}$. Fruits treated with potassium phosphite $\left(250 \mathrm{~mL} 100 \mathrm{~L}^{-1}\right)$ combined with $\mathrm{CaCl}_{2}(2 \%)$ had less decay and smallest lesion diammeter. These results were similar to those obtained with the fungicide Iprodione. Potassium phosphite alone was not as effective as in combination with $\mathrm{CaCl}_{2}$, and $\mathrm{Ca}+\mathrm{B}$ phosphite was not effective to control apple rot.

Key words: Malus domestica Borkh., leaf fertilizers, Penicillium spp.

\section{INTRODUÇÃO}

O uso de fungicidas químicos em pós-colheita vem sofrendo uma série de restrições, que recaem principalmente sobre o seu efeito residual, que pode restringir a exportação dos frutos e a sua comercialização. Atualmente, estão sendo pesquisadas formas alternativas para o controle de podridões pós-colheita de frutos, como a aplicação de produtos a base de fosfitos. Os fosfitos são compostos originados da neutralização do ácido fosforoso $\left(\mathrm{H}_{3} \mathrm{PO}_{3}\right)$ por uma base (hidróxido de sódio, hidróxido de potássio ou hidróxido de amônio). Esses compostos não são fitotóxicos e possuem elevada atividade fungicida (COHEN \& COFFEY, 1986). Sua ação sobre os fungos pode se dar de forma direta (FENN \& COFFEY, 1985; ROHRBACH \& SCHENCK, 1985) ou através da ativação de mecanismos de defesa da planta, como o estímulo à produção de fitoalexinas (GUEST \& GRANT, 1991; JACKSON et al., 2000). O tratamento com fosfitos induz a planta a apresentar resposta imediata ao ataque de patógenos (GUEST \& BOMPEIX, 1990).

Entre os resultados obtidos com a aplicação de fosfitos, WICKS et al. (1990) verificaram bons resultados no controle de míldio e doenças causadas

${ }^{1}$ Professor Adjunto, Doutor, Departamento de Fitotecnia, Universidade Federal de Santa Maria (UFSM), 97105-900, Santa Maria, RS. Email: brackmann@ccr.ufsm.br. Autor para correspondência.

${ }^{2}$ Acadêmico do Curso de Agronomia, UFSM. Bolsista do programa de Iniciação Científica (PROBIC), Fundação de Amparo à Pesquisa do Rio Grande do Sul (FAPERGS).

${ }^{3}$ Engenheiro Agrônomo, Mestrando do programa de Pós-graduação em Agronomia (PPGA), UFSM. Bolsista do Conselho Nacional de Pesquisa.

${ }^{4}$ Engenheiro Agrônomo, MSc, aluno do PPGA, UFSM. Bolsista CAPES. 
por Phytophthora spp., e GEELEN (1999) relatou resultados satisfatórios no controle de sarna e oídio em macieira. Na Austrália, o uso de fosfitos tem trazido bons resultados sobre o controle de Phytophthora cinnamomi em ecossistemas naturais (HARDY et al., 2001). No Brasil, VALDEBENITO-SANHUEZA (1991a; 1991b), verificou efeito positivo da aplicação de fosfitos na redução do desenvolvimento de lesões de Phytophthora cactorum em macieiras cv. Fuji enxertadas sobre porta-enxerto MM106 e inoculadas artificialmente. Em ensaios in vitro e testes preliminares realizados a campo, observaram-se resultados satisfatórios no controle da sarna da macieira (Venturia inaequalis), fuligem (Gloeodes pomigena) e sujeira-de-mosca (Schyzothyrium pomi) (BONETI \& KATSURAYAMA, 2002).

Não existem investigações sobre o efeito da aplicação de fosfitos em maçãs após a colheita. O objetivo deste trabalho foi avaliar os efeitos da aplicação de adubos foliares a base de fosfitos de potássio $(\mathrm{K})$ e de cálcio mais boro $(\mathrm{Ca}+\mathrm{B})$ sobre o controle de podridões em maçã 'Fuji'.

\section{MATERIAL E MÉTODOS}

O trabalho foi realizado no Núcleo de Pesquisa em Pós-Colheita (NPP) do Departamento de Fitotecnia da Universidade Federal de Santa Maria e utilizou maçãs 'Fuji' provenientes da empresa Gala Frigoríficos de Vacaria, onde estavam armazenadas por aproximadamente oito meses. O delineamento experimental utilizado foi o inteiramente casualizado com 7 tratamentos e 4 repetições compostas por 25 frutos cada. Após a homogeneização das amostras, foram feitas 4 lesões distribuídas de forma eqüidistante sobre a região equatorial dos frutos. As perfurações foram realizadas com o auxílio de uma ponteira de $3 \mathrm{~mm}$ de diâmetro e $5 \mathrm{~mm}$ de profundidade. Antes da aplicação de cada um dos tratamentos, os frutos permaneceram submersos por 20 segundos em uma solução com esporos de fungos em suspensão, provenientes de frutos com estruturas de Penicillium spp., Botrytis spp. e Rhizopus spp. Este processo foi realizado separadamente para cada tratamento, exceto o último, que teve o produto diluído na solução de esporos, no qual os frutos permaneceram por 1 hora. O objetivo disso foi simular o que ocorre, muitas vezes, em condições comerciais, quando os frutos são préresfriados em água altamente contaminada. Os demais tratamentos foram aplicados imergindo-se os frutos durante dois minutos em uma solução, contendo o respectivo tratamento. Os tratamentos aplicados foram: 1 - Testemunha (água); 2 - Iprodione $(75 \mathrm{~g}$ $\left.100 \mathrm{~L}^{-1}\right) ; 3$ - cloreto de cálcio $\left(\mathrm{CaCl}_{2}\right)$ a $2 \% ; 4$ - fosfito

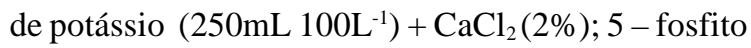

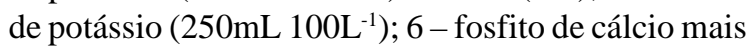

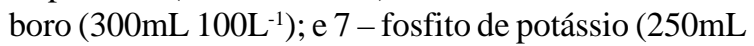
$\left.100 \mathrm{~L}^{-1}\right)$ na suspensão de esporos por uma hora. Os adubos foliares utilizados possuíam formulação 0040-20, para o caso do fosfito de potássio, e $11 \%$ de $\mathrm{P}_{2} \mathrm{O}_{5}, 3,9 \%$ de $\mathrm{Ca}$ e $0,5 \%$ de $\mathrm{B}$ na formulação do fosfito de $\mathrm{Ca}+\mathrm{B}$.

Depois da aplicação dos tratamentos, os frutos foram armazenados em uma câmara frigorífica à temperatura de $0^{\circ} \mathrm{C}$ durante 14 dias. Os frutos foram avaliados na saída da frigoconservação e aos 2, 4, 6 e 8 dias de exposição a $20^{\circ} \mathrm{C}$. Os parâmetros avaliados foram a porcentagem de lesões com desenvolvimento de podridão e a evolução das podridões, determinada pelo diâmetro das lesões através de uma régua milimetrada.

Os dados expressos em porcentagem foram transformados pela fórmula $\operatorname{arc}$.sen $\sqrt{x / 100}$ para serem, junto com os demais, submetidos à análise da variância. As médias foram comparadas entre si pelo teste de Duncan a 5\% de probabilidade de erro.

\section{RESULTADOS E DISCUSSÕES}

A incidência de podridões nos frutos na saída da câmara frigorífica foi nula em todos os tratamentos (dados não apresentados). No entanto, a exposição dos frutos a $20^{\circ} \mathrm{C}$ provocou um rápido desenvolvimento de fungos, principalmente após o $4^{\circ}$ dia . No $8^{\circ}$ dia, todas as lesões dos frutos do tratamento testemunha estavam podres. Os frutos

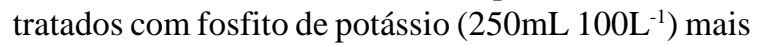
$\mathrm{CaCl}_{2}(2 \%)$ apresentaram porcentagem de lesões podres iguais estatisticamente aos frutos tratados com Iprodione aos 4, 6 e 8 dias de exposição a $20^{\circ} \mathrm{C}$. Esses resultados confirmam as afirmações de COHEN \& COFFEY (1986), que atribuíram aos fosfitos elevada ação fungicida. Além disso, os fosfitos podem estimular uma rápida produção de fitoalexinas, que são importantes mecanismos de defesa das plantas (NEMESTOTHY \& GUEST, 1990; GUEST \& BOMPEIX, 1990). A aplicação de

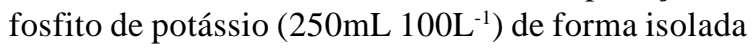
apresentou resultados significativamente inferiores à aplicação deste associado com $\mathrm{CaCl}_{2}(2 \%)$, principalmente no $6^{\circ}$ e $8^{\circ}$ dia de exposição à temperatura de $20^{\circ} \mathrm{C}$ (Tabela 1). Estes resultados revelam uma ação de sinergismo entre o fosfito de potássio e o cloreto de cálcio. $\mathrm{O}$ uso isolado de $\mathrm{CaCl}_{2}$ 
Tabela 1 - Efeito da aplicação de fosfitos de potássio e de cálcio mais boro sobre a porcentagem de lesões podres em maçãs 'Fuji'. Santa Maria, RS.

\begin{tabular}{|c|c|c|c|c|}
\hline Tratamentos & \multicolumn{4}{|c|}{ Dias a $20^{\circ} \mathrm{C}$} \\
\hline Testemunha & $47,5 a^{*}$ & $98,3 \mathrm{a}$ & $99,5 \mathrm{a}$ & $100,0 \mathrm{a}$ \\
\hline Iprodione $\left(75 \mathrm{~g} \mathrm{~L}^{-1}\right)$ & $1,0 \mathrm{~cd}$ & $23,0 \mathrm{e}$ & $61,3 \mathrm{~cd}$ & $76,0 \mathrm{c}$ \\
\hline Fosfito de potássio $\left(250 \mathrm{~mL} 100 \mathrm{~L}^{-1}\right)+\mathrm{CaCl}_{2}(2 \%)$ & $1,5 \mathrm{~cd}$ & $25,8 \mathrm{e}$ & $52,0 \mathrm{~d}$ & $70,8 \mathrm{c}$ \\
\hline 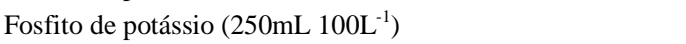 & $1,8 \mathrm{~cd}$ & $29,5 \mathrm{de}$ & $73,8 \mathrm{bc}$ & $88,5 \mathrm{~b}$ \\
\hline Fosfito de $\mathrm{Ca}+\mathrm{B}\left(300 \mathrm{~mL} 100 \mathrm{~L}^{-1}\right)$ & $3,3 \mathrm{c}$ & $81,3 \mathrm{c}$ & 97,0 a & $99,0 \mathrm{a}$ \\
\hline Fosfito de potássio $\left(250 \mathrm{~mL} 100 \mathrm{~L}^{-1}\right)$ na solução de esporos** & $0,3 \mathrm{~d}$ & $39,3 \mathrm{~d}$ & $81,0 \mathrm{~b}$ & $91,3 \mathrm{~b}$ \\
\hline
\end{tabular}

* Tratamentos com médias não seguidas pela mesma letra diferem estatisticamente pelo teste de Duncan a 5\% de probabilidade de erro.

** Os frutos ficaram submersos em uma solução contendo esporos em suspensão e o produto diluído nesta.

(2\%) não foi eficiente no controle de podridões (Tabela 1).

Observou-se que, mesmo quando os frutos permaneceram na solução de esporos durante 1 hora, a eficiência da aplicação de fosfito de potássio sobre a incidência de podridões foi estatisticamente igual à do tratamento com fosfito de potássio durante dois minutos, mostrando que sua ação fungicida é elevada até em condições extremas de contaminação (Tabela 1). MATHERON \& MATJKA (1988) verificaram que os fosfitos apresentam ação sistêmica podendo permanecer ativos por até 160 dias em citrus. Para HARDY et al. (2001), os fosfitos apresentam variabilidade considerável em relação à eficiência e persistência entre as mais diversas espécies de plantas, época e freqüência de aplicação.

A aplicação de fosfito de potássio associado com $\mathrm{CaCl}_{2}$ resultou em diâmetro de lesões iguais ao uso do fungicida Iprodione, demonstrando elevado efeito sobre a redução do desenvolvimento de podridões (Tabela 2). De acordo com BONETI \& KATSURAYAMA (2002), os fosfitos podem atuar de forma a reduzir fortemente o crescimento micelial, a formação de esporângios e a liberação de zoospóros, podendo reduzir a taxa de colonização de Phytophthora cinnamomi (ALI et al., 1998).

Os frutos tratados com fosfitos de $\mathrm{Ca}+$ B apresentaram elevada incidência de podridões,

Tabela 2 - Efeito da aplicação de fosfitos de potássio e de cálcio mais boro sobre o diâmetro das lesões (mm) em maçãs 'Fuji'. Santa Maria, RS.

\begin{tabular}{|c|c|c|}
\hline \multirow{2}{*}{ Tratamentos } & \multicolumn{2}{|c|}{ Dias a $20^{\circ} \mathrm{C}$} \\
\hline & 6 & 8 \\
\hline Testemunha & $24,1 a^{*}$ & $31,0 \mathrm{a}$ \\
\hline Iprodione $\left(75 \mathrm{~g} \mathrm{~L}^{-1}\right)$ & $14,0 \mathrm{~d}$ & $17,6 \mathrm{~d}$ \\
\hline $\mathrm{CaCl}_{2}(2 \%)$ & $22,8 \mathrm{~b}$ & 29,8 a \\
\hline Fosfito de potássio $\left(250 \mathrm{~mL} 100 \mathrm{~L}^{-1}\right)+\mathrm{CaCl}_{2}(2 \%)$ & $14,7 \mathrm{~d}$ & $19,4 \mathrm{~cd}$ \\
\hline Fosfito de potássio $\left(250 \mathrm{~mL} 100 \mathrm{~L}^{-1}\right)$ & $14,7 \mathrm{~d}$ & $18,5 \mathrm{~d}$ \\
\hline Fosfito de $\mathrm{Ca}+\mathrm{B}\left(300 \mathrm{~mL} 100 \mathrm{~L}^{-1}\right)$ & $19,8 \mathrm{c}$ & $26,2 \mathrm{~b}$ \\
\hline 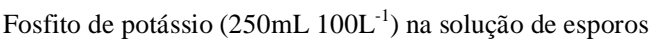 & $14,7 \mathrm{~d}$ & $20,4 \mathrm{c}$ \\
\hline Coeficiente de variação (\%) & 3,85 & 5,22 \\
\hline
\end{tabular}

* Tratamentos com médias não seguidas pela mesma letra diferem estatisticamente pelo teste de Duncan a 5\% de probabilidade de erro.

** Os frutos ficaram submersos em uma solução contendo esporos em suspensão e o produto diluído nesta. 
apresentando resultados iguais à testemunha a partir dos 6 e 8 dias de exposição a $20^{\circ} \mathrm{C}$ (Tabela 1). Em relação ao diâmetro das lesões, os frutos tratados com fosfito de $\mathrm{Ca}+\mathrm{B}\left(300 \mathrm{~mL} 100 \mathrm{~L}^{-1}\right)$ apresentaram lesões maiores que os demais, demonstrando que este tratamento não foi eficiente no controle e no desenvolvimento de podridões póscolheita em maçãs 'Fuji' (Tabela 2).

\section{CONCLUSÕES}

A aplicação de fosfito de potássio 0040-20 (250mL 100 $\left.\mathrm{L}^{-1}\right)$, associado ao $\mathrm{CaCl}_{2}(2 \%)$, apresentou resultados satisfatórios no controle de podridões pós-colheita em maçãs 'Fuji' frigoconservadas, podendo substituir o fungicida Iprodione (Rovral ${ }^{\circledR}$ ). Todavia, são necessárias novas investigações, visando a comparação de produtos comerciais com diferentes concentrações de fósforo e potássio de fabricantes distintos. Além disso, é necessário avaliar o efeito desses produtos após períodos prolongados de armazenamento.

\section{REFERÊNCIAS BIBLIOGRÁFICAS}

ALI, Z.; SMITH, I.; GUEST, D.I. Potassium phosphonate controls root rot of Xanthorrhoea australis and X. minor caused by Phytophthora cinnamomi. Australasian Plant Pathology, Orange, v.28, p.225-234, 1998.

BONETI, J.I; KATSURAYAMA, Y. Viabilidade do uso de fosfitos no manejo das doenças de macieira. In: ENCONTRO NACIONAL SOBRE FRUTICULTURA DE CLIMA TEMPERADO, 5., 2002. Fraiburgo, SC. Anais... Fraiburgo : EPAGRI, 2002. V.5, 307p., p.125-139.

COHEN, M.D.; COFFEY, M.D. Systemic fungicides and the control of oomycetes. Annual Review of Phytopathology, v.24, p.311-338, 1986.

FENN, M.E.; COFFEY, M.D. F urther evidence for direct mode of action of phosethyl-al and phosphorous acid. Phytopathology, St. Paul, v.75, p.1064-1068, 1985.
GEELEN, J.A. An evaluation of Agrio-Fos Supra 400 for the control of black spot and powdery mildew of apple in Hawke's Bay. N.I. :Geelen Research. Independent Horticultural Consulants, 1999. 15p.

GUEST, D.I.; GRANT, B.R. The complex action of phosphonates as antifungal agents. Biological Review, v.66, p.159-187, 1991.

GUEST, D.I.; BOMPEIX, G. The complex action mode of action of phosphonates. Australasian Plant Pathology, Orange, v.19, p.113-115, 1990.

HARDY, G.E. St.J.; BARRETT, S.; SHEARER, B.L. The future of phosphite as a fungicide to control the soilborne plant pathogen Phytophthora cinnamomi in natural ecosystems. Australasian Plant Pathology, Orange, v.30, p.133-139, 2001.

JACKSON, T.J. et al. Action of the fungicide phosphite on Eucaliptus marginata inoculated with Phytophthora cinnamomi. Plant Pathology, v.49, p.147-154, 2000.

MATHERON, M.E.; MATJKA, J.C. Persistence of systemic activity for fungicides applied to citrus trunk for control Phytophthora gummosis. Plant Disease, v.72, p.170-174, 1988.

NEMESTOTHY, G.S.; GUEST, D.I. Phytoalexin accumulation, phenylalamine ammonia lyase activity and ethylene biosynthesis in fosetil-al treated resistant and susceptible tobacco cultivars infected with Phytophthora nicotiana var. nicotianae. Physiological and Molecular Plant Pathology, v.37, p.207219,1990 .

ROHRBACH, K.G.; SCHENCK, S. Control of pineapple heart rot, caused by Phytophthora parasitica and P. Cinnamomi, with fosetyl-al and phosphorous acid. Plant Disease, v.69, p.320-323, 1985.

VALDEBENITO-SANHUEZA, R.M. Controle químico da podridão de raízes de macieira causada por Phytophthora cactorum no Rio Grande do Sul. Fitopatologia Brasileira, v.16, p.25-29, $1991 \mathrm{a}$.

VALDEBENITO-SANHUEZA, R.M. Tratamento de mudas de macieiras inoculadas com Phytophthora cactorum em condições de telado. Summa Phytopathologica, v.17, p.154-158, $1991 \mathrm{~b}$.

WICKS, T.J. et al. Evaluation del fosfito potasico como fungicida en Australia. Conferencia de Brinhton para protección de las cosechas. Pestes y Enfermedades, 1990. 